Tropical Journal of Pharmaceutical Research, June 2008; 7 (2): 937-944

(C) Pharmacotherapy Group,

Faculty of Pharmacy, University of Benin

Benin City, Nigeria.

All rights reserved.

Research Article

Available online at http://www.tjpr.org

\title{
Evaluation of Adverse Drug Reactions to Artemisinin- based Combination Therapy in a Nigeria University Community
}

\author{
R Adisa*, TO Fakeye and D Dike
}

Department of Clinical Pharmacy \& Pharmacy Administration, University of Ibadan, Ibadan, Nigeria.

\begin{abstract}
Purpose: The study was carried out to evaluate the incidence of adverse reactions to antimalarial drugs among residents of a Nigeria university community with a focus on artemisinin-based combination therapy (ACT). Specifically, the profile of use, and the reporting culture of people with respect to experienced reactions were noted.

Method: Questionnaires were administered to respondents at the university health centre between November 2006 and January 2007. Information on demographic characteristics, nature of experienced adverse reactions and the most frequently used ACT, among other questions, were collected. Descriptive statistics and Fisher's Exact test were used to evaluate the distribution of respondent's opinion.

Result: The study achieved a response rate of $86 \%$. The results revealed that 210 (70.0\%) of respondents said they had used artemisinin-based combination drugs while 134 (44.7\%) said they used artemisinin derivatives alone as monotherapy for malaria treatment. Artesunate plus amodiaquine 94 (31.3\%) as a co-packaged product was the most commonly used ACT. Incidence of the experienced adverse reactions to ACT was reported to be generally mild and well tolerated.

Conclusion: Efforts to improve the use of ACT in the management of acute uncomplicated $P$. falciparum malaria is recommended. Furthermore, an effective mechanism to improve reporting of adverse effects of ACT is also recommended
\end{abstract}

Keywords: Adverse Drug Reaction, Artemisinin- combination therapy (ACT), malaria

“Corresponding author: Email: adisaras73@yahoo.co.uk \& rasaq.adisa@mail.ui.edu.ng Tel: 2348034226199 


\section{INTRODUCTION}

In many Africa countries, intensity of malaria transmission is high and antimalarial drugs are used frequently for presumptive treatment of fever, even in the absence of laboratory confirmed malaria diagnosis ${ }^{1,2,3,4,5}$. Informal use of antimalarial drugs may increase the risk of incorrect dosing, inappropriate treatment, occurrence of adverse drug reactions and interaction of different medicines, which could have a negative impact on antimalarial treatment safety $5,6,7,8$. Increasing parasite resistance and failure of single drug treatment of malaria in many endemic countries of Africa $^{9}$ has led to a widespread promotion of artemisinin-based combination therapy (ACT) as a strategy for effective management of Plasmodium falciparum malaria ${ }^{10}$. World Health Organization (WHO) has, since 2001, recommended that malaria endemic countries change their treatment policies and adopt combination therapy, and in particular artemisinin-based combination therapy as the first- line antimalarial treatment ${ }^{11}$. Artemeterlumefantrine, artesunate (AS) plus amodiaquine (AQ), artesunate plus sulphadoxine/pyrimethamine (SP), and artesunate plus mefloquine are some of the ACT that have been recommended for use in Africa based on available efficacy and safety data $^{11,12 .}$

The current plans for a wide- scale implementation of ACT across Africa offer an opportunity to evaluate the occurrence of adverse drug reactions to these medicines when used in the larger population or the community. Gabon and Sao Tome had implemented the use of artesunate plus amodiaquine, with report of minor side effects while Burundi and Zanzibar reported that the safety of ACT for the last two years had been good $^{1,12}$. Other Africa countries are at various stages of preparations to implement chosen ACT. China, Thailand and South East Asia are also some of the countries that have used artemisinin-based combination therapy extensively with no report of any serious adverse reactions ${ }^{13}$.
Nigeria has successfully adopted the use of artemisinin-based combination therapy for malaria treatment for more than two years with WHO recommended drug-combinations available in the country under different proprietary names. To the best of our knowledge, there are no substantial reports of incidence of adverse effects associated with the use of these medicines aside those documented during clinical trials or premarketing phase. However, obtaining information on adverse reaction profile of ACT will be necessary in countries (such as Nigeria) where self-treatment with antimalarial drugs is pervasive. This is to ensure that the common and rare adverse drug events to ACT are documented and, also to ensure that patients are getting the desired therapeutic benefits rather than negative consequences on account of such adverse drug effects.

To detect such adverse events, monitoring the use and evaluating patients' response to antimalarial drugs, particularly the new combination therapies, outside of clinical trials at the health facilities and community levels becomes essential ${ }^{14,15,16}$. It is in line with this background that this study was carried out to evaluate the incidence of adverse drug reactions to antimalarial drugs among residents of a Nigerian university community with a focus on artemisinin-based combination therapy (ACT). Information on the profile of use and reporting culture of people with respect to experienced adverse reactions to ACT were also obtained.

\section{METHODS}

The study area, University of Ibadan, is the oldest university community in the country. It has all the necessary infrastructure and amenities that can be found in a typical community including offices and housing accommodation for staff, their families and students of the institution. The study site, the university health centre, has in its employment 10 physicians, 5 pharmacists, 22 nurses, 2 physiotherapists, 13 laboratory technologists and other ancillary health workers. It is also the only clinic within the university 
environment that caters for the health care needs of the entire university community.

The study design was cross-sectional and involved the use of structured questionnaires administered to the patients at the study site within a period of three months (November 2006-January 2007). The questionnaire was designed to have two sections: the first section contained questions on demographic characteristics of respondents while the second section contained open- and closedended questions to obtain relevant information on the experienced adverse reactions to antimalarial drugs, particularly ACTs. Information was also obtained on the profile of use, adverse drug reaction reporting culture of respondents, among other questions. The questionnaire was pre-tested among five experienced heath care workers (3 pharmacists and 2 doctors) in the locality for content validity and these individuals were subsequently not included in the study.

Permission to conduct the study was obtained from the authorities of the University Health Services and approval to conduct the study at the centre was granted. Subjects who presented at the study site with symptoms suggestive of malaria fever or history of recent fever were approached for participation in the study, after they had seen their physicians. The objective of the study was explained to the subjects and those who were interested in the study consented to participate. Informed verbal consent was obtained from individual subject at every contact. Excluded from the study were children, pregnant women, and unconscious patients. Participating subjects were assured of their anonymity.

Three hundred and fifty questionnaires were administered directly to the subjects at the study site within the three-month period. Each participant was given between 20 and 30 minutes to fill in their responses. Only the 300 questionnaires $(85.7 \%)$ that were properly filled were subjected to statistical analysis.

Data generated were analyzed using Graph Pad InStat ${ }^{\circledR}$ 3. Fisher's Exact Test was used to determine whether there was an association between certain variables and some of the responses, while descriptive statistics were used to evaluate the distribution of respondent opinions to other questions. level significance (p) was set at 0.05 .

\section{RESULTS}

Three hundred questionnaires which were properly filled were analysed. There were 160 $(53.3 \%)$ male and 140 (46.7\%) female respondents, out of which, $225(75.0 \%)$ were single while 75 (25.0\%) were married. Respondents in the age group of 21-30 years constituted the largest proportion 172 (57.3\%), while only $8.7 \%$ were 41 years and above; those in the age groups $12-20$ and $31-40$ years constituted 55 (18.3\%) and $47(15.7 \%)$, respectively. Opinion on the frequency of use of antimalarial drugs showed that a majority, 127 (42.3\%) had used antimalarial medication for presumptive treatment of fever, 100 $(33.3 \%)$ took these drugs only when it was recommended in the hospital, while 48 $(16.0 \%)$ claimed to have taken antimalarial drugs occasionally for prophylactic treatment of malaria.

One third of the respondents, 102 (34.0\%), believed that they have malaria whenever there is high body temperature; 94 (31.3\%) mentioned joint pains and loss of appetite as the symptoms that prompt them to selftreatment with antimalarial drugs. Eighty-four $(28.0 \%)$ said they do not take antimalarial drugs until physician's diagnosis indicate malaria illness, while only 20 (6.7\%) claimed that they do not treat malaria until they undertake laboratory confirmation for malaria parasites. The frequency profile of antimalarial drugs used by respondents is shown in Table 1.

Two-thirds of the respondents, 206 (68.7\%), said they have access to artemisinin-based combination therapy since these medicines are readily available for purchase in most pharmacies and patent medicine shops, while $25(8.3 \%)$ believed that both the branded and generic products of artemisinin-based combination drugs are not accessible because they are expensive and unaffordable. Fifty $(16.7 \%)$ respondents would not allow the high cost of ACTs to discourage them from 
purchasing the drug as long as they find the drug effective.

Out of the total number of respondents 210 $(70.0 \%)$ that had used artemisinin-based combination drugs (namely artesunate plus amodiaquine, arthemether-lumefantrine, artesunate plus sulphadoxine/pyrimethamine, and artesunate plus mefloquine), 69 (32.9\%) had experienced one form of reaction or the other with the administered ACT, while 137 $(65.2 \%)$ had not. The experienced reactions, summarized in Table 2, were mainly some of the common adverse effects documented in literature to be associated with the corresponding ACT. Respondents found these reactions tolerable.

Itching or pruritus was experienced by only $0.3 \%$ of the respondents that took artesunate plus amodiaquine and artesunate plus mefloquine, whereas twenty-two percent experienced needle-prick sensation with chloroquine monotherapy. Rashes were completely absent with most respondents that used ACT except with $0.7 \%$ of the respondents who combined artesunate with sulphadoxine/pyrimethamine, a reaction that was also experienced by $3.3 \%$ of respondents on sulphadoxine/pyrimethamine monotherapy. Incidence of vomiting and abdominal pain was more with artemeter-Imefantrine $1.7 \%$ and $4.0 \%$ respectively, while $1.3 \%$ and $2.3 \%$ of the respondents that took artesunate plus amodiaquine experienced nausea and abdominal pain respectively.

General body weakness was experienced by respondents who combined artesunate with amodiaquine (5.3\%), artemeter-lumefantrine $(2.7 \%)$, artesunate plus mefloquine and artesunate plus sulphadoxine/pyrimethamine had $2.3 \%$ each. Body weakness was also experienced by $5.0 \%$ of respondents on chloroquine monotherapy. Ear and eye disturbances were experienced by $1.3 \%$ and $1.0 \%$ of the respondents that took artesunate plus amodiaquine and artemeter-lumefantrine respectively.

A majority, 52 (75.4\%), of those that experienced adverse reactions with ACTs did not report the reactions to any health care professional. The reasons given for not reporting were that most of the reactions were tolerable and some respondents were not knowledgeable on how and where to report such reactions. However, 17 (24.6\%) of those that experienced adverse reactions claimed to have reported these reactions to a health care provider and appropriate advice were given based on the reaction experienced.

Fisher's Exact statistical test showed that sex did not appear to have any significant relationship with the incidence of experienced adverse reactions to the ACTs used $(P=0.25)$ (Table 3)

\section{DISCUSSION}

More than two-third of the respondents in this study had used ACT, which is probably an indication of increased awareness by the people on the use of ACT for treatment of uncomplicated falciparum malaria. However, almost half of the respondents used antimalarial drugs including ACT for selftreatment of malaria indicating irrational use of the antimalarial agents. Such an informal use of drugs may increase the risk of drug problems and consequently, could have a negative impact on the continued effectiveness and safety of antimalarial treatment ${ }^{5,6,7,8}$.

Despite the usefulness of artemisinin derivatives, its use as monotherapy should not be encouraged due to the possibility of emergence of resistance strains to the single drug treatment on repeated and inappropriate use ${ }^{9,10,17}$. Close to half of the respondents in this study were found using artemisinin derivatives as monotherapy, a practice that may lead to the development of resistant strains. There is a need to encourage patients to combine an artemisinin derivative with another antimalarial drug that has a longer half life such as amodiaquine, lumefantrine, sulphadoxine/pyrimethamine and mefloquine, in order to ensure complete eradication of malaria parasites and allow for a reduction in the duration of antimalarial treatment $^{10,18,19,20}$.

Artesunate plus amodiaquine was found to be the most commonly used ACT despite some of the adverse drug reactions experienced 
Adisa et al

Table 1: Frequency profile of antimalarial drugs used by respondents

\begin{tabular}{lcc}
\hline Drugs & Frequency & $\begin{array}{c}\text { Percentage } \\
\text { (\%) }\end{array}$ \\
\hline Artesunate plus Amodiaquine & 94 & 31.3 \\
Artemeter- lumefantrin & 47 & 15.7 \\
Artesunate plus & 41 & 13.7 \\
Sulphadoxine/pyrimethamine & & \\
Artesunate plus mefloquine & 28 & 9.3 \\
Artesunate alone & 134 & 44.7 \\
Sulphadoxine/pyrimethamine & 202 & 67.3 \\
Chloroquine & 166 & 55.3 \\
Amodiaquine & 35 & 11.7 \\
Halofantrin & 5 & 1.7 \\
\hline
\end{tabular}

such as general body weakness, ear and eye disturbances, abdominal pain and dizziness. Some of these reactions could have been due to the amodiaquine component of the combination. The preference of artesunate combined with amodiaquine by most people for the treatment of malaria may probably be due to availability of several branded products of artesunate plus amodiaquine in the country and its relatively low cost when compared with artemeter-lumefantrine.

Itching/pruritis which has been known to be one of the worrisome side effects of chloroquine and sometimes amodiaquine rarely occurred in most respondents that took ACT. This is encouraging since needle-prick sensation is one of the side effects that discourage patient adherence to treatment using 4-aminoquinoline antimalarial agents. Rash was not found to occur in most respondents that took artemisinin-based combination drugs except for a few of the respondents that took artesunate plus sulphadoxine/pyrimethamine, a reaction which was probably due to the sulphadoxine/pyrimethamine component of the combination.

General body weakness, blurred vision or dizziness which were experienced by some respondents that took artesunate plus amodiaquine, could also have been due to the amodiaquine component since some of the documented side effects of 4-aminoquinoline antimalarial agents includes dizziness, general weakness, blurred vision and fatigue ${ }^{12}$.

Artemeter-lumefantrin recorded the highest number of respondents with incidence of abdominal pain. Abdominal pain has been documented in literature as one of the bothersome side effects of artemeterlumefantrine that may affect adherence ${ }^{21}$, though none of the respondents claimed to have stopped the use of this combination because of abdominal pain.

It is encouraging to note that though most of the respondents had experienced incidences of adverse drug reactions with the use of ACT, the general inference that could be drawn from their responses was that they found the reactions tolerable. There was no report of life-threatening adverse drug reactions that could warrant termination of treatment or drug use. This has confirmed reports from China, Thailand, South East Asia and other Africa countries where ACTs have been used extensively and were found to be relatively safe and well tolerated ${ }^{5,6,7,8}$.

Most respondents that experienced adverse reactions with ACT did not report those reactions to any health care professional. However, a few that did were not given any adverse drug reactions (ADR) form to fill but were given appropriate counsel. This finding indicates the poor reporting habit of ADR by health care providers ${ }^{22}$, and is one of the issues that need to be addressed by the body in charge of pharmacovigilance. 
Table 2: Adverse drug reactions experienced by respondents and the antimalarial drugs implicated

\begin{tabular}{|c|c|c|c|c|c|c|c|c|c|c|c|c|}
\hline DRUGS & 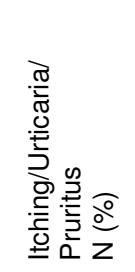 & 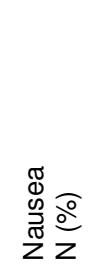 & 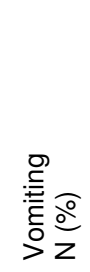 & 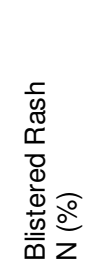 & 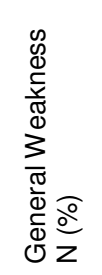 & 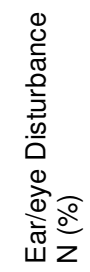 & $\begin{array}{l}\frac{0}{0} \\
\frac{0}{0} \\
\frac{\pi}{0} \\
\mathbb{d} \\
\frac{\mathbb{d}}{1} z\end{array}$ & 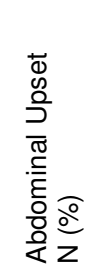 & $\begin{array}{l}\mathscr{D} \\
\stackrel{\mathscr{C}}{N} \widehat{0} \\
\stackrel{N}{0} Z\end{array}$ & $\begin{array}{l}\frac{\mathbb{d}}{\Phi} \\
\frac{c}{\frac{\tau}{ \pm}} \\
\frac{0}{0} \\
\frac{0}{2}\end{array}$ & 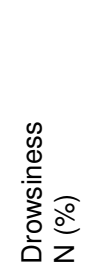 & 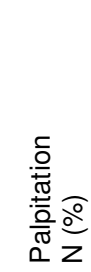 \\
\hline Chloroquine & $65(21.7)$ & $3(1.0)$ & $1(0.3)$ & - & $15(5.0)$ & $4(1.3)$ & $6(2.0)$ & - & $13(4.3)$ & - & $1(0.3)$ & - \\
\hline $\begin{array}{l}\text { Sulphadoxine/pyrim } \\
\text { ethamine }\end{array}$ & $3(1.0)$ & $4(1.3)$ & $1(0.3)$ & $10(3.3)$ & $13(4.3)$ & - & $3(1.0)$ & $1(0.3)$ & $7(2.3)$ & $1(0.3)$ & $2(0.7)$ & $1(0.3)$ \\
\hline Amodiaquine & $1(0.3)$ & $3(1.0)$ & $3(1.0)$ & - & $8(2.7)$ & - & $2(0.7)$ & - & $1(0.3)$ & - & - & $1(0.3)$ \\
\hline $\begin{array}{l}\text { Artesunate } \\
\text { (as monotherapy) }\end{array}$ & $1(0.3)$ & $3(1.0)$ & $2(0.7)$ & - & $10(3.3)$ & $1(0.3)$ & $2(0.7)$ & - & $6(2.7)$ & - & $1(0.3)$ & - \\
\hline $\begin{array}{l}\text { Artesunate }(A S)+ \\
\text { Amodiaquine }(A Q)\end{array}$ & $1(0.3)$ & $4(1.3)$ & $3(1.0)$ & - & $16(5.3)$ & $4(1.3)$ & $6(2.0)$ & $7(2.3)$ & $5(1.7)$ & - & $1(0.3)$ & - \\
\hline $\begin{array}{l}\text { Artemeter- } \\
\text { lumefantrin }\end{array}$ & - & $1(0.3)$ & $5(1.37$ & - & $8(2.7)$ & $3(1.0)$ & $5(1.7)$ & $12(4.0)$ & $3(1.0)$ & $1(0.3)$ & - & $3(1.0)$ \\
\hline $\begin{array}{l}\text { Artesunate + } \\
\text { Mefloquine }\end{array}$ & $1(0.3)$ & $1(0.3)$ & $2(0.7)$ & - & $7(2.3)$ & $1(0.3)$ & $3(1.0)$ & $3(1.0)$ & $3(1.0)$ & $1(0.3)$ & - & - \\
\hline $\begin{array}{l}\text { Artesunate }+ \\
\text { Sulphadoxine/pyrim } \\
\text { ethamine }\end{array}$ & - & $3(1.0)$ & $1(0.3)$ & $2(0.7)$ & $7(2.3)$ & $1(0.3)$ & $1(0.3)$ & $4(1.3)$ & $1(0.3)$ & - & - & $3(1.0)$ \\
\hline
\end{tabular}


Table 3: Fisher's Exact Test to show the relationship between sexes and respondent's experience of adverse reactions to artemisinin based combination therapy (ACT).

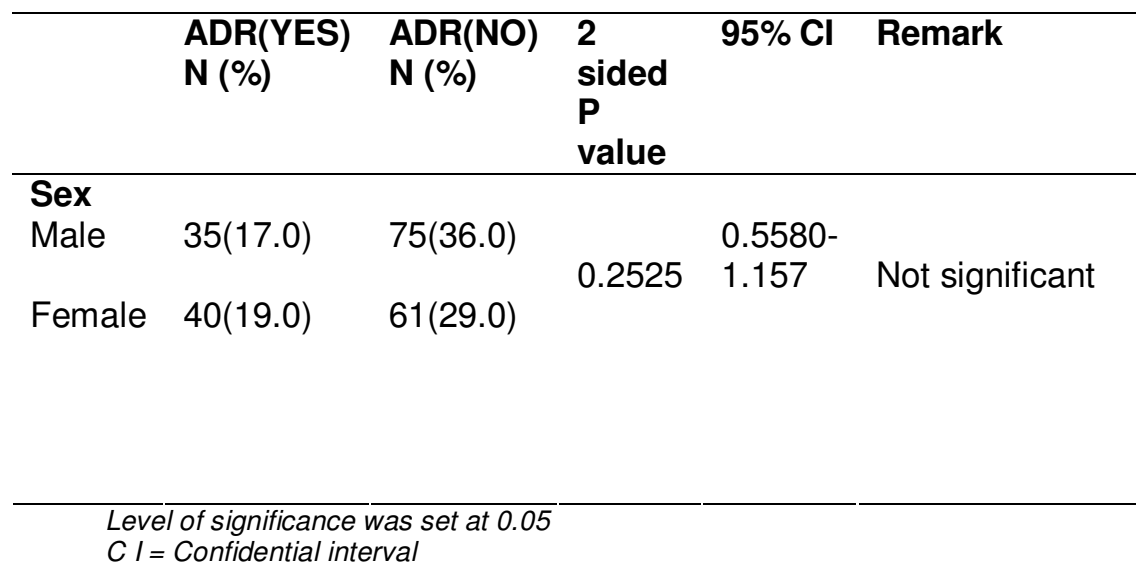

\section{CONCLUSION}

The incidence of experienced adverse reactions to the ACTs used for malaria treatment in this study was found to be generally mild and tolerable. Sex did not appear to have any significant effect on the incidence of experienced adverse reactions to ACT. Reporting of the experienced ADR by the affected patients was generally low while subsequent reporting to the pharmacovigilance centers by healthcare providers was non-existent. Effort should be made to encourage the use of ACT in the management of acute uncomplicated $P$. falciparum malaria. Furthermore, institutionalization of an effective mechanism to improve the reporting of adverse effects of ACT is recommended. A future study to assess the safety of this group of antimalarial drugs in vulnerable populations should, therefore, be considered in order to generate a useful safety information and database to improve the quality of health care offered to the patient.

\section{ACKNOWLEDGEMENT}

We are grateful to Professor OA Itiola for his useful suggestions and comments after reading.

\section{REFERENCES}

1. Mclntosh $H M$, Olliaro $P$. Artemisinin derivatives for treating uncomplicated malaria. Cochrane Database syst Rev 2000 CD 000256.

2. Taylor $W R$, White $N$ J. Antimalarial drug toxicity: a review. Drug saf 2004; 27:25-61

3. White $N \mathrm{~J}$, Olliaro P. Artemisinin and derivatives in the treatment of uncomplicated malaria. Med Trop 1998; 58 (suppl):54-56.

4. Adjuik M, Babiker A, Garner P, Olliaro P, Taylor W, White N. International Artemisinin Study Group. Artesunate combination for the treatment of malaria; meta- analysis. Lancet 2004; 363:917.

5. Talisuna $O A$, Sarah G, Umberto D'Alessandro. Pharmacovigilance of antimalarial treatment in Africa; Is it possible. Malaria Journal 2006; Jun16; 5(1):50

6. Rogier $C$,Tall A, Diagne $N$, Fontenelle $D$, Spiegel $A$, Trape JF. Plasmodium falciparum clinical malaria: lession from Longitudinal studies in Senegal. Parassitologia 1999; 41:255-59.

7. Dorsey G, Njama $D$, Kamya $M R$, Rosenthal P. A longitudinal assessment of different first-line therapies for uncomplicated falciparum malaria in Kampala, Uganda. $50^{\text {th }}$ Annual meeting of the America Society of Tropical Medicine and Hygiene 2001, Atlanta, Georgia.

8. Olliaro $P$, Nevil $C$, LeBras J et al. Systematic review of amodiaquine treatment in Uncomplicated Malaria. Lancet 1996; 1196-201

9. Trape JF. Impact of chloroquine resistance of malaria mortality. Comptes rendu de L'Academie des sciences 1998; 321 (Series 111):689-697.

10. Lyda O, Iveth G, Piero $O \&$ Walt $R$ J. Artemisininbased combination therapy for uncomplicated Plasmodium falciparum malari.a in Colombia. Malaria Journal 2007, 6:25 
11. WHO: Guidelines for the treatment of malaria. Geneva, World Health Organization; 2006.

12. Abdullah A, Moh'd O. Pharmacovigilance in Zanzibar; Current status. Pharmacovigilance Unit-Zanzibar National Malaria Control Programme. The Newsletter for the East African Network for monitoring antimalarial treatment (EANMAT) volume 8, issue2 sept.2005.

13. Hellgren $U$, Berggreb-palme, Bergqvis $Y$, Jerling $M$. Enantioselective pharmacokinetics of mefloquine during long term intake of the prophylactic dose. Br. J. Clin. Pharmacol. 1997; 44: 119-1

14. Breman J. The ears of the hippopotamus: manifestations determinants and estimates of the malaria burden. Am J Trop Med Hyg 2001; 64:1-11.

15. Philips- Howard PA, West L J. Serious adverse drug reactions to pyrimethamine-sulphadoxine, pyrimethamine- dapsone and to amodiaquine in Britain. Journal of the Royal Society of Medicine 1990; 83:82-5

16. Price R. Adverse effects in patients with acute falciparum malaria treated with artemisinin derivatives. Am J Trop Med Hyg 1999; 60:547555.
17. Erah P.O, Avienmughare $G$ \& Okhamafe $O A$. Plasmodium falciparum resistance to chloroquine in five communities in Southern Nigeria. Africa Journal of Biotechnology 2003:2 (10):384-389.

18. World Health Organization. The Importance of pharmacovgilance safety monitoring of Medicinal products. Geneva, 2002.

19. Heppner DG, Ballou WR. .Malaria in 1998: Advances in diagnosis, drug and vaccine development. Tropical and Travel Assoc. Dis. 1998; 519-518.

20. Salako L.A, Ajayi F.O, Sowunmi $A$ \& Walker O. Malaria in Nigeria: A Revisit. Ann Trop Med and Parasitol, 1990; 8:435-444.

21. Simooya OO, Sijumbil G, Lennard MS, Turker G. Halofantrine and chloroquine inhibit Cy2D6 activity in healthy Zambians. Br.J. Clin. Pharmacol.1998; 45: 315-317

22. Fakeye T, Onyemadu O. Evaluation of knowledge base of hospital Pharmacists and physicians on herbal medicines in Southwestern Nigeria. Pharmacy Practice (www. Pharmacypractice. org) 2008 (In press). 